\title{
Clinical and radiologic outcomes after a modified bone plug technique with anatomical meniscal root reinsertion for meniscal allograft transplantation and a minimum 18-month follow-up
}

\author{
Shiyou Ren ${ }^{1+}$, Xintao Zhang ${ }^{1,2+}$, Tian You ${ }^{1}$, Xiaocheng Jiang ${ }^{1}$, Dadi Jin ${ }^{2}$ and Wentao Zhang ${ }^{1 *}$
}

\begin{abstract}
Objective: To evaluate the clinical and radiologic outcomes of meniscal allograft transplantation (MAT) using a modified bone plug technique.

Methods: We conducted a retrospective single-center study of 73 patients who underwent MAT between January 2007 and December 2013. The International Knee Documentation Committee (IKDC) score, Lysholm score, Tegner score, visual analogue scale (VAS), and physical examinations were retrospectively reviewed to measure clinical outcomes after MAT, and questionnaires regarding activity and factors were analyzed. Magnetic resonance imaging (MRI) was used to assess the cartilage status and meniscal extrusion.

Results: The mean follow-up was 37 months for 61 patients (65 knees), and 12 patients were lost to follow-up. The mean meniscal extrusion was $3.39 \pm 0.90 \mathrm{~mm}$, the relative percentage of extrusion (RPE) was $34.82 \% \pm 12.71 \%$, and arthrosis progression was observed in 8 of 61 cases (13.1\%). The mean results for VAS, IKDC, and Lysholm scores were significantly improved after MAT $(P<0.05)$, but there were no significant differences in the range of motion or Tegner score $(P>0.05)$. Thirty-eight (62.3\%) patients were able to return to their previous level of activity, and 23 (37.7\%) patients reached a mean $76.7 \%$ of the previous level of activity. Of the 23 patients reporting a decrease in activity, 10 reported a fear of reinjury as the primary factor limiting activity. The patient satisfaction rate in the study was $78.7 \%$.
\end{abstract}

Conclusion: Our modified bone plug method with anatomical meniscal root reinsertion was an effective surgical method, and the majority of active patients with meniscal disorders returned to preinjury levels of activity.

Keywords: Meniscal allograft transplantation, Modified bone plugs, Knee, Arthroscopy

\section{Introduction}

The meniscus plays an essential role in the function and biomechanics of the knee joint, providing an even load distribution across the joint, thereby decreasing peak contact forces on the tibiofemoral articular cartilage [1]. Meniscus tears are one of the most common injuries in sports medicine and may result either from acute knee

\footnotetext{
* Correspondence: zhangwt2007@sina.cn

${ }^{\dagger}$ Equal contributors

${ }^{1}$ Department of Sports Medicine and Rehabilitation, Peking University Shenzhen Hospital, 1120 Lianhua Road, Futian District, Shenzhen 518036, Guangdong, China

Full list of author information is available at the end of the article
}

trauma or through degenerative processes. The management of meniscal tears is varied and often dependent on the severity of the injury, including nonoperative treatment, meniscectomy, repair, and transplantation [2]. In cases of irreparable meniscus tears or meniscal deficiency after meniscectomy, there may be considerable pain and there is a high risk of developing degenerative disease of the knee joint over time. How to restore the function of the meniscus to induce an early regenerative progress has increasingly been recognized as a clinical challenge. Since the meniscal transplantation procedure was first described by Milachowski et al. in 1984 [3], meniscal 
allograft transplantation (MAT) has become a viable option in meniscus-deficient patients.

However, MAT remains a controversial treatment for meniscus-deficient patients [4] because of its underlying conditions of uncertainty, such as whether living-donor tissue is required for optimal attachment, the long-term outcomes, whether it can delay the degenerative progress, and whether movement should be restricted postoperatively. Fortunately, an increasing number of authors have attempted to develop this procedure and have reported good results. However, the use of bone plugs is still controversial. Certain studies have achieved good clinical and biomechanical results with bone plugs [5-7]. However, good clinical results using only suture fixation have also been described $[8,9]$. Furthermore, it is essential to ensure a precise size match between the graft and the host in techniques involving bone plugs, which can increase the risk of cartilage degeneration and incorrect positioning [7]. It can reduce the morbidity of surgery with suture fixation and can be performed under arthroscopy. In contrast, it is technically easier to perform fixation with soft tissue alone, but research has shown that the load distribution is superior when the allograft is secured with bone $[10,11]$. Therefore, we used an arthroscopic double tibial tunnel technique for MAT with new modified bone plugs to ensure initial fixation and sound bone-to-bone healing in a consecutive series of symptomatic patients with a previous total or subtotal meniscectomy [12]. The purpose of this study was to evaluate the clinical and radiologic outcomes of MAT using a new modified bone plug technique with anatomical meniscal root reinsertion.

\section{Patients and methods}

\section{Study overview}

This study and procedure were approved by our institutional review board. From January 2007 to December 2013, 73 consecutive patients underwent MAT. Of these, 61 patients ( 36 men and 25 women) with a mean age of 32.3 years were followed up for more than 18 months and were enrolled in this study. The followup rate was $83.6 \%$, and the mean follow-up duration was 31.0 months (18-80 months).

Indications for surgery included irreparable tears diagnosed preoperatively and during arthroscopy or persistent symptoms after meniscectomy, normal alignment or correction-to-normal alignment, and a stable ligamentous knee condition or correction to a stable ligamentous knee condition. Five out of 61 patients underwent immediate MAT without any chondral injury or symptoms after meniscectomy, and the remaining patients underwent delayed MAT with symptoms of pain or decreased range of motion (ROM) after meniscectomy.

Preoperative radiographic assessment was performed on all patients including the following: weight bearing anteroposterior and 3D CT of the knee, a lower extremity examination and MRI. The irreparable meniscal tears were diagnosed by MRI preoperatively and confirmed by arthroscopy. The occurrence and degree of osteochondral injury were assessed by MRI using the Outerbridge grade. Meniscal deficiency was confirmed by a history of arthroscopic meniscectomy and MRI.

There were 21 left knees and 35 right knees with lateral MAT, 3 knees with medial and lateral MAT and 6 right knees with medial MAT. Four patients underwent lateral MAT of both knees. Not all of the patients received meniscal transplantation alone. Some patients were found to have concomitant anterior cruciate ligament (ACL) injuries, cartilage injury, MCL injuries, or contralateral meniscus injury in the same knee; therefore, additional orthopedic procedures- medial collateral ligament reconstruction (MCLR), anterior cruciate ligament reconstruction (ACLR), and MF-were performed in some cases, and for one patient, osteotomy was performed to address coronal malalignment of the lower limbs. Additional procedures performed at the time of MAT are included in Table 1. The mean time from the total meniscectomy to the secondary MAT was 36.6 weeks. Three patients underwent a lateral MAT, combined medial MAT and ACLR at the same time. These procedures showed the advantages of the new technique in that little space is required to fix the anterior or posterior horns of the meniscus, and both lateral and medial MATs were secured on the tibial simultaneously without damaging the tibial insertion of the PCL or encroaching on the sites for tibial tunnels of ACLR.

\section{Surgical procedure}

The sizing protocol was based on the 3D CT reconstruction measurements [13] combined with matching of the height, weight and sex between the donor and recipient [14]. In all cases, fresh-frozen, irradiated menisci were used (Fig. 1). All procedures were performed by a single surgeon (Wentao Zhang).

The arthroscopy-assisted MAT was performed with a bone plug technique [15] for MAT. A 4-mm diameter spherical anterior bone plug and a 4-mm diameter spherical posterior bone plug size (approximately the size of a grain of rice) were cored from the meniscal allograft to permit sound bone-to-bone healing with the tibia. Ethibond (No. 5; Ethicon, Somerville, NJ) was placed with a whipstitch

Table 1 Combined operation with meniscal allograft transplantation in all 61 cases

\begin{tabular}{lllllll}
\hline & ACLR & Microfx & OT & MCLR & CMSK & ORIF \\
\hline L-MAT & 7 & 9 & 2 & 1 & 1 (MAT) & 1 \\
M-MAT & 3 & 2 & 0 & 0 & 1 (repair) & 0 \\
L/M-MAT & 3 & 0 & 0 & 0 & 0 & 0 \\
\hline
\end{tabular}



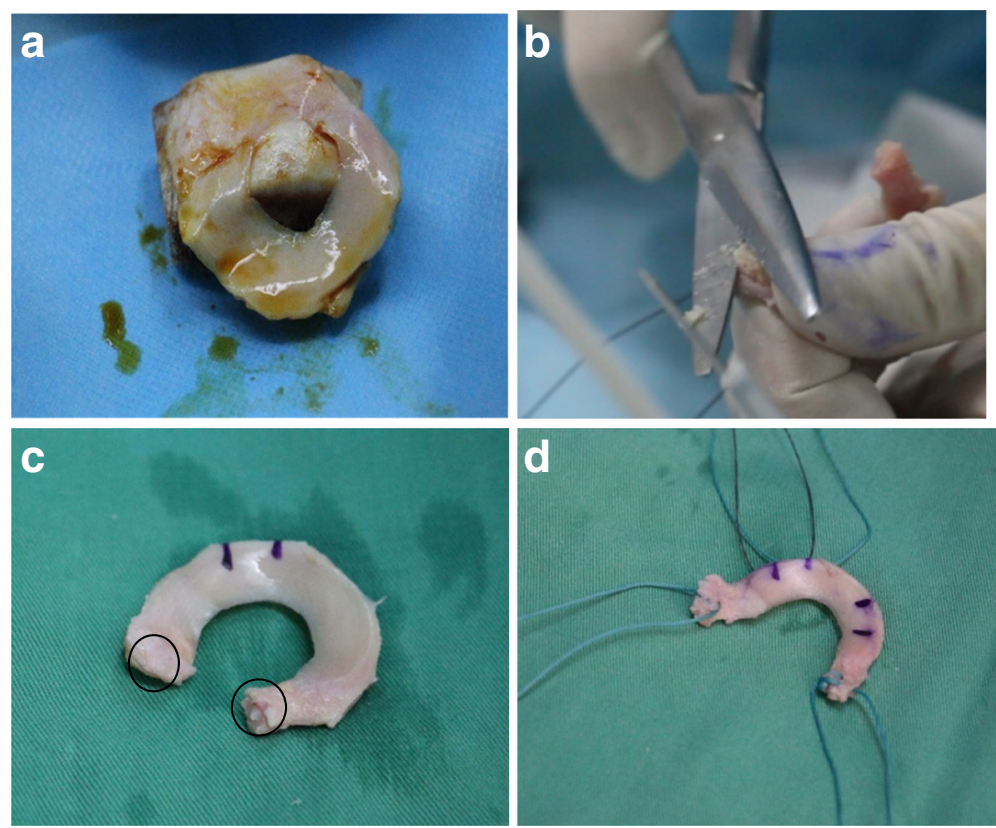

Fig. 1 Trimmed meniscal allograft. a Fresh-frozen, irradiated menisci. b The bone plug was thinned with scissors. c Allograft with bone plug (the bone plug in the black circle is $4 \mathrm{~mm}$ in diameter) was marked with radial signs with a surgical marker. $\mathbf{d}$ Ethibond (No. 5; Ethicon, Somerville, NJ) was placed with a whipstitch at each horn for later traction, spreading, and securing the horn of the allograft

in each horn for later traction and spreading. The allograft was marked with radial signs with a surgical marker to prevent mismatching and twisting during arthroscopic insertion. The meniscus remnant was shaved until the meniscus-capsular zone was reached. A tibial guide, a $2.0-\mathrm{mm}$ guide drill and a $4.5-\mathrm{mm}$ core drill were used to prepare two tibial tunnels through which the Ethibonds corresponding to each horn of the menisci were placed. A knot pusher with perforation at one end was used as needed to pass the two Ethibonds through the bone tunnels (Fig. 2).

Position of bone tunnels: the medial meniscus posterior horn inserts directly anterior to the tibial insertion of the PCL, on the downslope of the posterior intercondylar fossa, behind the posterior horn insertion of the lateral meniscus. The posterior horn of the lateral meniscus inserts directly posterior to the lateral tibial spine, adjacent and anterior to the insertion of the posterior horn of the medial meniscus [16]. The anterior horn of the medial meniscus inserts in line with the medial tibial eminence, approximately $7 \mathrm{~mm}$ anterior to the ACL tibial insertion. This insertion site is under the patellar fat pad and is difficult to visualize without debriding a portion of the anterior fat pad. The intermeniscal ligament attaches to the posterior half of this insertion site. The insertion site of the anterior horn of the lateral meniscus is directly anterior to the lateral tibial spine and adjacent to the tibial insertion of the ACL (Fig. 3).
Suturing was started from posterior to anterior in order to firmly attach the graft to the joint capsule. All sutures from the body of the meniscus were tied to the capsule. The posterior horn area, which did not receive any stitch, was attached to the posterior capsule with Fast-Fix (S\&N, Andover, MA, USA) all-inside sutures (Fig. 4). Before suturing, the correct placement of the graft was checked. The lower suture should be removed before the upper one. When all sutures are outside the knee, they can be sutured to the capsule. If additional stitches are necessary to ensure a stable graft, this stitch must be out-in. Finally, the sutures from the anterior and posterior tunnels were sutured to each other, leaving both meniscus horns anchored [8]. Then, the transplanted meniscus was checked for stability and matching (Fig. 5).

The mean time of the operation was $2.05 \mathrm{~h}$. Of the transplants, 13 were performed concomitant with ACL reconstruction.

\section{Rehabilitation}

Early ROM for 15 min daily in the range of $0^{\circ}$ to $60^{\circ}$ was encouraged beginning 1 week postoperatively to minimize the deleterious effects of immobilization. Gradual progression to full weight bearing occurred by 6 weeks postoperatively. A double upright hinged brace was used during this designated protection phase. Flexion was limited to 90 for the first 6 weeks, as progressive knee flexion subjects the meniscus to greater stress [9]. Full ROM was achieved by 


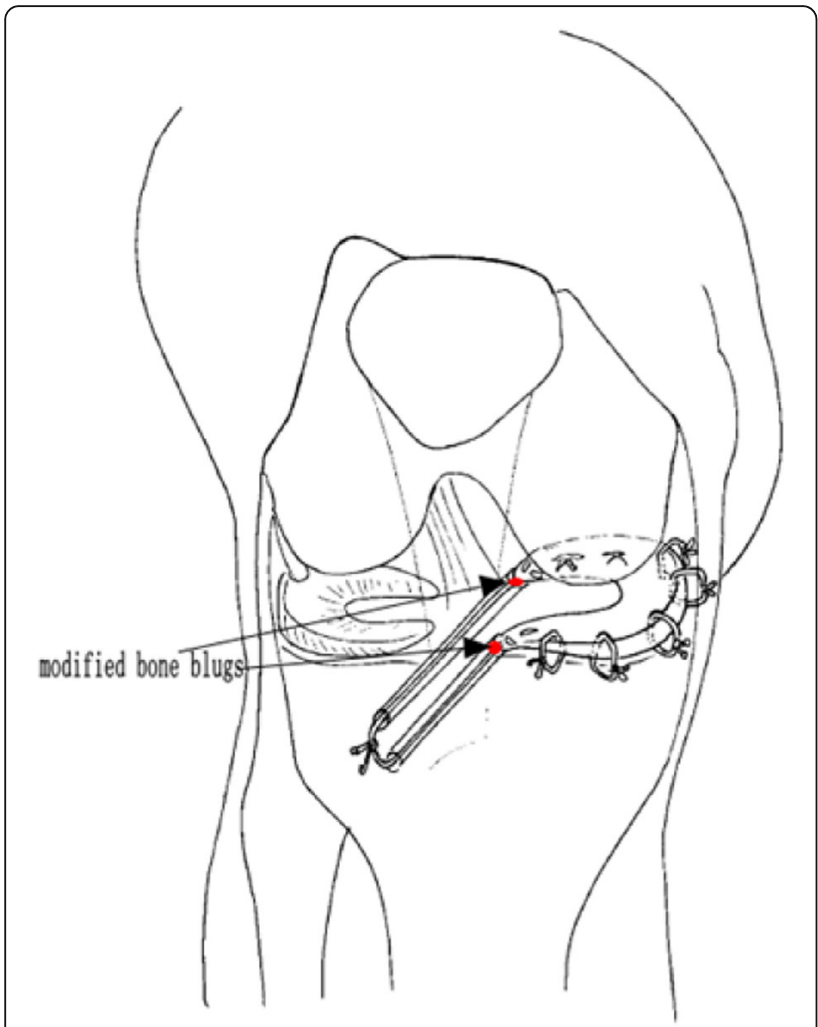

Fig. 2 Schematic drawing of the presented arthroscopic modified bone plug technique using double tibial tunnels for meniscal allograft transplantation. Red points mark the bone plugs, and the modified bone plugs were placed in the bone tunnels to permit sound bone-to-bone healing with the tibia

14 weeks postoperatively. Gentle jogging could begin between 4 and 6 months, but running was not advised before 6 months post-surgery. Finally, return to sports was anticipated to occur between 6 and 9 months [7]. In cases of concomitant ACL reconstruction, the rehabilitation protocol was modified according to the surgeon's concern for both the ACL and the meniscus. Training to strengthen muscles and improve proprioception was conducted throughout the rehabilitation protocol.

\section{Follow-up}

All patients were followed up for more than 18 months postoperatively for ROM, International Knee Documentation Committee (IKDC) score, Lysholm score, Tegner score, and VAS for pain. Physical examinations and associated measurements were repeated postoperatively. Physical examination and radiographic parameters during follow-up were available in all cases. Patients were asked two series of questions: 1 . Were you able to return to your previous level of activity? (Yes/No). If yes, what sports could you do? Can you do a single-leg jump? If no, what degree from 0 to $100 \%$ according to the visual analogue scale after surgery did you reach compared with your previous level of activity and what is the main factor limiting the activity? 2. Based on your experience, would you have the motivation to seek the same treatment in case of being subjected to the same injury on the contralateral knee? (Yes/No/Unsure). All responses were collected and recorded by a single observer. The intraobserver reliability of this observer ranged from moderate to excellent for all parameters tested. We evaluated cartilage status according to the modified Outerbridge classification system. MRI examinations were performed on a $3 \mathrm{~T}$ cylinder-shaped instrument. Orthopedic surgeons measured meniscal extrusion to the nearest millimeter on the coronal images and obtained mean values and standard deviation. The relative percentage of meniscal extrusion was defined as the percentage of extruded meniscal width compared with the entire meniscal width [17].

\section{Statistical analysis}

Statistical analysis was performed with SPSS software for Windows (version 19.0, SPSS Inc., Chicago, IL, USA). The data were tested via the normality test (Shapiro-Wilk test,

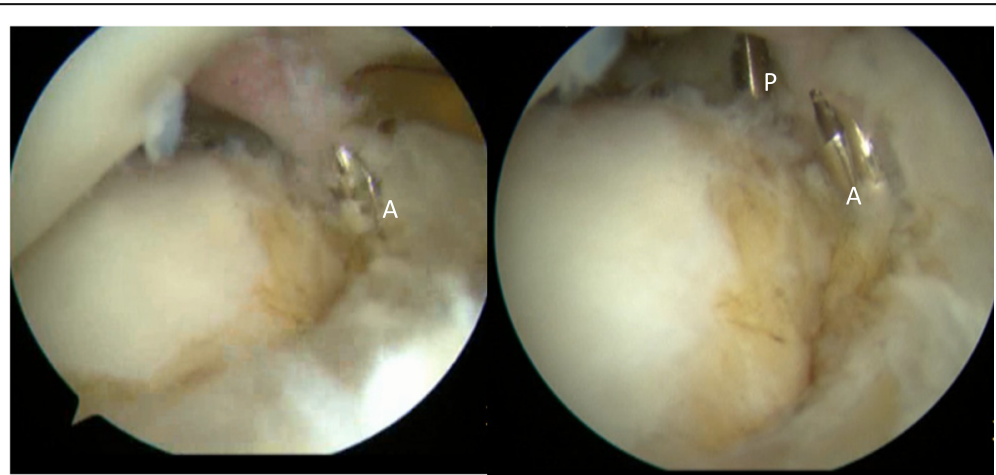

Fig. 3 A tibial guide and a 2.0-mm guide drill were used to locate the attachment points of the meniscus posterior horn and anterior horn. A presents the insertion site of the anterior horn of the lateral meniscus, while P presents the insertion site of the posterior horn insertion of the lateral meniscus 

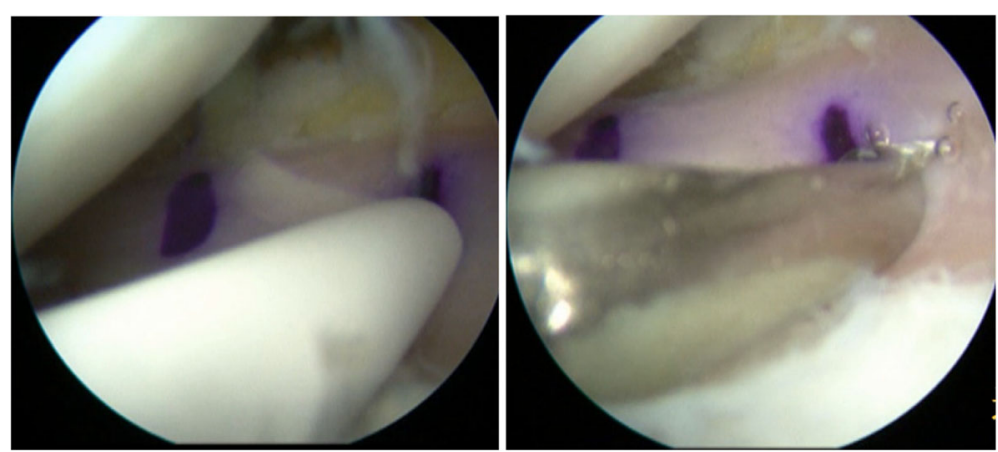

Fig. 4 The posterior horn area, which has not received any stitches, is attached to the posterior capsule with Fast-Fix all-inside sutures

$n<2000)$ and did not follow a Gaussian distribution. The Wilcoxon rank sum test was used to compare preoperative and postoperative IKDC score, Lysholm score, Tegner score, VAS, cartilage status and to correlate the influence of associated procedures and previous lesions. Data on meniscal extrusion and RPE were analyzed. Spearman correlation analysis was used to determine whether the changes in MAT follow-up results were associated with possible risk factors, including age at time of MAT, meniscal extrusion, the time from the total meniscectomy to the secondary MAT. $P<0.05$ was considered significant.

\section{Results}

Cartilage status was evaluated by MRI according to the modified Outerbridge system. The mean meniscal extrusion was $3.39 \pm 0.90 \mathrm{~mm}$, the relative percentage of extrusion (RPE) was $34.82 \pm 12.71 \%$, and arthrosis progression was observed in 8 of 61 cases (13.1\%), 6 of which also underwent ACLR. The Wilcoxon rank sum

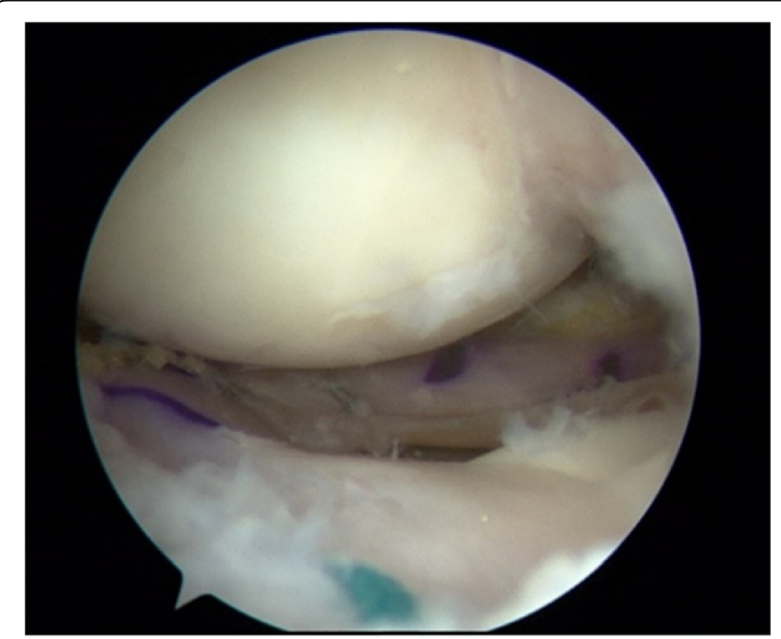

Fig. 5 Final arthroscopic view of an implanted medial meniscal allograft in the left knee of a female 35-year-old patient test showed there was no significant difference between preoperative and postoperative cartilage status (Table 2), and there was no significant difference in MAT follow-up results between different preoperative cartilage status groups (Table 3). We found significant association between the changes in postoperative IKDC score (positive correlation, $P<0.05$ ), Lysholm score (positive correlation, $P<0.05$ ), Tegner score (positive correlation, $P<0.05$ ), VAS (negative correlation, $P<0.05$ ), and the time from the total meniscectomy to the secondary MAT and meniscal extrusion $(P<0.05)$, no significant association between the changes in MAT follow-up results and age $(P>0.05)$ (Table 4).

The mean results for postoperative VAS, IKDC score, and Lysholm score were significantly better than the data for preoperation $(P<0.05$, Wilcoxon test), while there was no significant difference in the ROM and Tegner score pre- and postoperation $(P>0.05$, Wilcoxon test) (Table 5).

Comparing the outcome scores between lateral and medial transplantation revealed no significant difference in (ROM), IKDC score, Lysholm score, Tegner score, or VAS $(P>0.05$, Wilcoxon test) (Table 6).

Comparing the outcome scores between MAT, MAT $+\mathrm{MF}$, and MAT+ACLR revealed no significant difference in IKDC score, Lysholm score, Tegner score, or VAS $(P>0.05$, Wilcoxon test) (Table 7). Only 3 patients with MAT+MF + ACLR, 2 with OT, 1 with MCLR, and 1 with open

Table 2 Cartilage status on MRI

\begin{tabular}{lll}
\hline Arthrosis grade on MRI & Preoperatively & Postoperatively \\
\hline 0 & 8 & 7 \\
1 & 17 & 13 \\
2 & 23 & 25 \\
3 & 7 & 8 \\
4 & 6 & 8 \\
$P$ Value & $Z=-0.911$ & $P=0.36$ \\
\hline
\end{tabular}


Table 3 Comparisons of MAT follow-up results between different preoperative cartilage status groups (Wilcoxon test)

\begin{tabular}{llllll}
\hline $\begin{array}{l}\text { Arthrosis grade on MRI } \\
\text { (preoperatively) }\end{array}$ & $N$ & Post-op VAS & Post-op IKDC & Post-op Tegner & $\begin{array}{l}\text { Post-op Lysholm } \\
P_{50}\left(P_{25}-P_{75}\right)\end{array}$ \\
\hline 0 & 8 & $4(3.25,5.75)$ & $78(70.5,87)$ & $4.5(4,5.75)$ & $81.5(76.5,85)$ \\
1 & 17 & $3(3,4)$ & $82(79,85.5)$ & $6(5,6)$ & $83(82,86.5)$ \\
2 & 23 & $3(3,4)$ & $84(81,87)$ & $6(5,6)$ & $87(84,89)$ \\
3 & 7 & $3.5(3,4)$ & $85(80,88)$ & $5(5,6)$ & $85(75,89)$ \\
4 & 6 & $4(2.75,5)$ & $85(82,86.75)$ & $5.5(5,6)$ & $85.5(83,88.75)$ \\
$H$ & & $H=5.4, P=0.249$ & $H=4.6, P=0.331$ & $H=7.746, P=0.101$ & $H=7.56, P=0.109$ \\
\hline
\end{tabular}

$P 50$ median, $P 75-P 25$ interquartile range)

reduction internal fixation (ORIF), there was no reliable statistical analysis to correlate the influence of these associated procedures because of smaller number.

With regard to the postoperative examination of the patients who underwent MAT combined with ACL reconstruction, the anterior drawer test and Lachman test were negative. All X-rays and MRI of the 51 patients showed no joint space narrowing. According to the Stoller standard, with regard to reinjury of the transplanted meniscus, 13 showed no reinjury, $25 \mathrm{I}^{\circ}$ reinjury, $17 \mathrm{II}^{\circ}$ reinjury and $6 \mathrm{III}^{\circ}$ reinjury at the 18-month follow-up. All of the meniscus formed a union with the capsule confirmed by MRI. The modified bone plugs were inserted into the bone tunnels to form sound bone-tobone healing with the tibia 2 years after meniscal allograft transplantation (Fig. 6). One incision showed delayed union combined with fat liquefaction. Two patients who underwent concomitant ACL reconstruction developed synarthrophysis. One suffered a postoperative joint infection. There was one failure of the operation and one meniscus allograft dislocation, treated with a second operation for meniscal restoration.

Thirty-eight $(62.3 \%)$ patients were able to return to their previous level of activity, and 25 of them returned to football or basketball, 2 returned to tennis, 3 returned to table tennis, 5 returned to badminton, and 3 returned to Kung fu; however, in 3 of these cases, the medial side of the knee was slightly painful during a single-leg jump. The remaining 23 patients reached a mean $76.7 \%$ of the previous level of activity. Of the $23(37.7 \%)$ patients reporting a decrease in activity, 10 reported the fear of reinjury as the primary factor limiting activity. Of the remaining patients, pain $(n=5)$, limitation of ROM $(n=4)$, medical advice $(n=3)$, and a change in life situation $(n=1)$ were the reported primary reasons for decreased activity.

Asked if they would choose to undergo the procedure again in case of being subjected to the same injury on the contralateral knee based on their experience, 42 (68.9\%) patients stated that they would, 4 cases (6.5\%) said they would refuse the possibility of the same treatment, and 15 (24.6\%) were unsure about their decision.

\section{Discussion}

During this research, cartilage status was evaluated by MRI according to the modified Outerbridge system, and the Wilcoxon rank sum test showed there was no significant difference between preoperative and postoperative cartilage status. Arthrosis progression was observed in 8 of 61 cases (13.1\%). Among these patients, 6 cases were reinjured after MAT, and there was one failure of the operation and one meniscus allograft dislocation, and there was no significant difference in MAT follow-up results between different preoperative cartilage status groups. It has been speculated that MAT with the new modified bone plug technique with anatomical meniscal root reinsertion could delay the progression of arthrosis. This conclusion is in accordance with Bum-Sik Lee's result-"Articular cartilage degenerates after subtotal/total lateral meniscectomy but radiographic arthrosis progression is reduced after meniscal transplantation" [18] —and is in line with prior research using a bone plug technique $[17,19]$. In addition, there was significant association

Table 4 The changes in MAT follow-up results associated with possible risk factor (Spearman correlation analysis)

\begin{tabular}{lllll}
\hline & Post-op VAS & Post-op IKDC & Post-op Tegner & Post-op Lysholm \\
\hline Age & Pearson $=0.03$, & Pearson $=-0.034$, & Pearson $=-0.054$, & $P$ Pearson $=-0.028$, \\
& $P=0.821$ & $P=0.798$ & $P=0.678$ & $P=0.831$ \\
The time from the total meniscectomy & $P$ earson $=-0.342$, & $P$ Pearson $=0.559$, & $P$ Pearson $=0.467$, & $P$ Pearson $=0.565$, \\
to the secondary MAT & $P=0.08$ & $P=0.002$ & $P=0.014$ & $P=0.002$ \\
Meniscal extrusion & $P$ Pearson $=0.323$, & $P$ Pearson $=-0.286$, & $P$ Pearson $=-0.235$, & $P$ Pearson $=-0.263$, \\
& $P=0.011$ & $P=0.026$ & $P=0.068$ & $P=0.041$ \\
\hline
\end{tabular}


Table 5 Comparisons of preoperative and postoperative data in all 61 cases (Wilcoxon test)

\begin{tabular}{|c|c|c|c|c|c|c|c|}
\hline \multirow[t]{2}{*}{ Items } & \multirow[t]{2}{*}{$N$} & \multicolumn{2}{|c|}{ Pre-op } & \multicolumn{2}{|c|}{ Follow-up } & \multirow[t]{2}{*}{ W } & \multirow[t]{2}{*}{ P } \\
\hline & & $P_{50}$ & $P_{75}-P_{25}$ & $P_{50}$ & $P_{75}-P_{25}$ & & \\
\hline VAS & 61 & 5 & 3 & 3 & 1 & 1478.5 & $<0.001$ \\
\hline IKDC & 61 & 68 & 15 & 84 & 6 & 146 & $<0.001$ \\
\hline Tegner & 61 & 5 & 2 & 6 & 1 & 467 & 0.062 \\
\hline Lysholm & 61 & 69 & 13 & 85 & 5 & 141 & $<0.001$ \\
\hline ROM & 61 & 145 & 10 & 145 & 10 & 709 & 0.122 \\
\hline
\end{tabular}

$P 50$ median, $P 75-P 25$ interquartile range)

between MAT follow-up results and the time from the total meniscectomy to the secondary MAT, when the time from the previous meniscectomy to MAT increased, the follow-up results of MAT got worse.

Wang et al. [20] found that transosseous fixation at the meniscal horns provides superior load distribution in the involved knee compartment after meniscal transplantation compared with suture-only fixation. To a certain extent, this finding showed our modified technique was reliable. However, unlike traditional bone plug or bone bridge techniques, the modified technique requires little space to fix the anterior or posterior horns of the meniscus and does not damage the tibial insertion of the PCL and ACL. Almost all of the allografts demonstrated sound bone-to-bone healing with the tibia 2 years after MAT, in contrast to Roberson's soft tissue in bone socket fixation technique [21].

The mean meniscal extrusion was $3.39 \pm 0.90 \mathrm{~mm}$, and the relative percentage of extrusion (RPE) was $34.82 \% \pm$ $12.71 \%$ in our study, which is slightly superior to the traditional bone plug technique, whose mean meniscal extrusion and the relative percentage of extrusion were reported to be $4.35 \pm 1.76 \mathrm{~mm}$ and $43 \% \pm 19.8 \%$, respectively [19]. This difference might be due to racial differences or technique, but there is not enough evidence to be sure. And we found significant association between the changes in MAT follow-up results and meniscal extrusion, and it

Table 6 Comparison of outcome scores between lateral and medial transplantation (Wilcoxon test)

\begin{tabular}{|c|c|c|c|c|c|c|}
\hline \multirow[t]{2}{*}{ Items } & \multicolumn{2}{|c|}{$\begin{array}{l}\text { Medial MAT }(N=6) \\
\text { Follow-up-pre-op }\end{array}$} & \multicolumn{2}{|c|}{$\begin{array}{l}\text { Lateral MAT }(N=52) \\
\text { Follow-up-pre-op }\end{array}$} & \multirow[t]{2}{*}{ W } & \multirow[t]{2}{*}{ P } \\
\hline & $P_{50}$ & $P_{75}-P_{25}$ & $P_{50}$ & $P_{75}-P_{25}$ & & \\
\hline VAS & -2 & 2 & -2 & 2 & 202.5 & 0.521 \\
\hline IKDC & 21 & 7 & 13.5 & 14.75 & 313.5 & 0.108 \\
\hline Tegner & 1 & 1 & 0 & 2.25 & 303.5 & 0.154 \\
\hline Lysholm & 22 & 10 & 15 & 10.75 & 310 & 0.124 \\
\hline ROM & 0 & 15 & 0 & 16.25 & 265 & 0.529 \\
\hline
\end{tabular}

$P 50$ median, $P 75-P 25$ interquartile range) was different from Ji Hyun Ahn's [22] that there were no significant difference in Lysholm score and Tegner activity scale between minor extrusion group and major extrusion group after MAT.

In our patients, including cases with additional orthopedic procedures, MATs were performed simultaneously or in a staged manner along with ligament surgery such as ACLR or MF occurred most frequently. We conducted a subgroup analysis of both isolated MAT, MAT procedures with concomitant ligament surgery, and MAT with MF. And there were no significant difference in them at minimum 18-month follow-up, and the results were in accordance with Bum-Sik Lee [23]. With only three patients with MAT+MF+ACLR, two with OT, one with MCLR, and one with ORIF, there was no reliable statistical analysis to correlate the influence of these associated procedures because of the smaller number.

In addition, of the $23(37.7 \%)$ patients reporting a decrease in activity, 10 patients reported fear of reinjury as the primary factor limiting activity. We found in many cases that excessive flexion was limited compared with that of the contralateral knee. However, rehabilitation of the last angles of excessive flexion is somewhat difficult and painful, and most patients accepted the $5^{\circ}$ limitation and refused to continue the rehabilitation. A systematic review reported that MAT allows return to the same level of competition in $75-85 \%$ of patients in the short- to mid-term follow-up [24], the same as in our results. A recent systematic review [25] reported that 70-92\% of patients returned to a wide variety of sports activities, and there was no association between mean Tegner scores and transplant failure rates, but a moderate correlation was found between failure rates and mean follow-up time $(R=0.63)$. An analysis of the effect of return to high-impact activities on transplant failure rates or progression of OA was not possible because of the short-term follow-up.

Patients who do not resume sports primarily cannot return to their previous sports for two reasons: first, the allograft may prevent certain motions, and second, the rehabilitation protocol may have been conducted poorly. The use of a rehabilitation protocol that is appropriate for the surgical technique results in optimal postoperative outcomes and functional recovery of patients to a preinjury level of activity. The ideal rehabilitation program is based on biological and mechanical knowledge of the meniscus. Rehabilitation programs following meniscal transplantation are dependent on surgical technique, concomitant procedures, and pathology as well as surgeons' preference [26]. Rehabilitation protocols must strike a balance between maintaining joint motion and muscle strength and protecting the graft to allow full healing of the meniscus and any associated procedures [27]. To achieve this, most authors limit weight bearing and range of motion for up to 6 weeks 
Table 7 Comparisons of MAT follow-up results between different associated surgery procedures (MAT, MAT+MF, and MAT+ACLR) (Wilcoxon test)

\begin{tabular}{llllll}
\hline Procedure & $N$ & Post-op VAS & Post-op IKDC & Post-op Tegner & $\begin{array}{l}\text { Post-op Lysholm } \\
P_{50}\left(P_{25}-P_{75}\right)\end{array}$ \\
\hline MAT & 38 & $3.5(3,4)$ & $P_{50}\left(P_{25}-P_{75}\right)$ & $P_{50}\left(P_{25}-P_{75}\right)$ & $85(82,87)$ \\
ACL+MAT & 10 & $3.5(3,5)$ & $84(79.75,86.25)$ & $6(5,6)$ & $84(75.5,90)$ \\
MF+MAT & 8 & $3(2.75,3.25)$ & $80(76,85.5)$ & $5.5(4,6.25)$ & $84.5(77.25,89.25)$ \\
$H$ & $H=3.378, P=0.185$ & & & $5.5(79.75,88)$ & $H=0.079, P=0.961$ \\
\hline
\end{tabular}

before progressively allowing the resumption of activities. Rehabilitation protocols are divided into "traditional", involving restricted range of motion, weight bearing and improving muscle strength, and "aggressive", involving an intensive physiotherapy program, especially kinesiotherapy (achieving full extension immediately after surgery and early implementation of therapy combining concentric and eccentric contraction for the thigh muscle and exercise in the open kinetic chain). Our rehabilitation protocol belongs to the group of "traditional" rehabilitation protocols.

To the best of our knowledge, our study is the largest to report the outcomes of MAT using a modified bone plug technique with anatomical meniscal root reinsertion in China, and this study is unique. In this series, we have shown that the majority of patients return to their preinjury level of sports after this procedure. As with most procedures, the patients must be made fully aware of the uncertain effect of MAT on the eventual progression of articular cartilage degeneration and deterioration or traumatic disruption of their meniscus grafts. Longer term of follow-up should be continued to provide additional information.

This study has certain limitations. First, further details regarding the specific type and competitive level of pre- and postoperative sport and activity for each patient are not available for this cohort. Second, as a successful return to play may take up to 2 years after surgery, the follow-up should exclude those with less than 2 years following surgery. Finally, it is difficult to draw any final and definite conclusions. Despite these limitations, this retrospective cohort provides updated results regarding a new MAT technique in China.

\section{Conclusion}

Our modified bone plug method with anatomical meniscal root reinsertion is an effective surgical method, and the majority of active patients with meniscal disorders returned to preinjury levels of activity.

\section{Abbreviations}

ACL: Anterior cruciate ligament; ACLR: Anterior cruciate ligament reconstruction; CMSK: Contralateral meniscus in the same knee; IKDC: International Knee Documentation Committee; L/M-MAT: Lateral and medial MAT concomitantly; L-MAT: Lateral MAT; MAT: Meniscal allograft transplantation; MCLR: Medial collateral ligament reconstruction; Microfx: Microfracture; M-MAT: Medial MAT; MRI: Magnetic resonance imaging; ORIF: Open reduction internal fixation; OT: Chondral transplantation PM: Partial meniscectomy; RPE: Relative percentage of extrusion; VAS: Visual analogue scale

\section{Acknowledgements}

The authors thank Li Xiang and Sun Limang for collecting the participants' data.

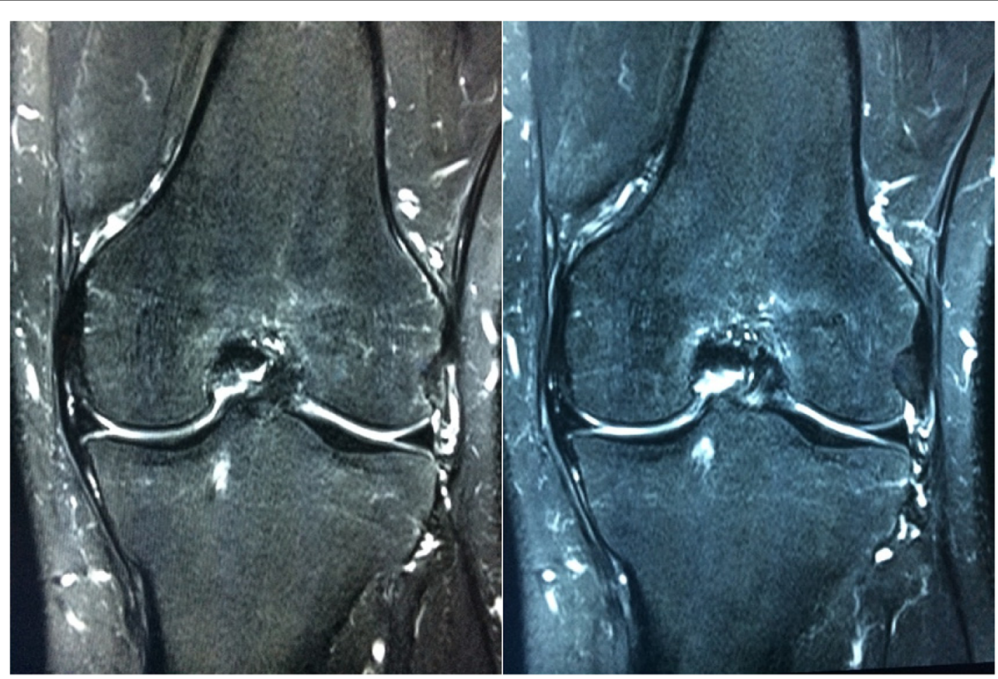

Fig. 6 The modified bone plugs were inserted into the bone tunnels to achieve sound bone-to-bone healing with the tibia 2 years after MAT 


\section{Funding}

This research received no specific grant from any funding agency in the public, commercial, or not-for-profit sectors.

\section{Availability of data and materials}

The rest of the data are available from the corresponding author upon reasonable request.

\section{Authors' contributions}

SYR and XTZ drafted the manuscript and revised it critically for important intellectual content. SYR and XTZ contributed equally to the work. TY performed statistical analysis. JXC collected the clinical data, and WTZ contributed substantially to the conception and design of the study. DDJ revised the manuscript. All authors read and approved the final manuscript.

\section{Ethics approval and consent to participate}

This study was approved by the ethics committee of Peking University Shenzhen Hospital. All patients provided informed consent.

\section{Consent for publication}

Not applicable.

\section{Competing interests}

The authors declare that they have no competing interests.

\section{Publisher's Note}

Springer Nature remains neutral with regard to jurisdictional claims in published maps and institutional affiliations.

\section{Author details}

'Department of Sports Medicine and Rehabilitation, Peking University Shenzhen Hospital, 1120 Lianhua Road, Futian District, Shenzhen 518036, Guangdong, China. ${ }^{2}$ Department of Orthopaedics, The Third Affiliated Hospital of Southern Medical University, Guangzhou, Guangdong, China.

Received: 29 October 2017 Accepted: 22 March 2018

Published online: 24 April 2018

\section{References}

1. Verdonk R, Volpi P, Verdonk P, Van der Bracht H, Van Laer M, Almqvist KF, et al. Indications and limits of meniscal allografts. Injury. 2013;44(Suppl 1): S21-7.

2. Kaleka CC, Debieux P, da Costa Astur D, Arliani GG, Cohen M. Updates in biological therapies for knee injuries: menisci. Curr Rev Musculoskelet Med. 2014;7:247-55

3. Milachowski KA, Weismeier K, Wirth CJ. Homologous meniscus transplantation. Experimental and clinical results. Int Orthop. 1989;13:1-11.

4. Hutchinson ID, Moran CJ, Potter HG, Warren RF, Rodeo SA. Restoration of the meniscus: form and function. Am J Sports Med. 2014;42:987-98.

5. Rijk PC. Meniscal allograft transplantation_-part I: background, results, graft selection and preservation, and surgical considerations. Arthroscopy. 2004; 20:728-43.

6. Rodeo SA. Meniscal allografts - where do we stand? Am J Sports Med. 2001;29:246-61.

7. Sekaran SV, Hull ML, Howell SM. Nonanatomic location of the posterior horn of a medial meniscal autograft implanted in a cadaveric knee adversely affects the pressure distribution on the tibial plateau. Am J Sports Med. 2002;30:74-82.

8. Alentorn-Geli E, Seijas Vázquez R, García Balletbó M, Álvarez Díaz P Steinbacher G, Cuscó Segarra X, et al. Arthroscopic meniscal allograft transplantation without bone plugs. Knee Surg Sports Traumatol Arthrosc. 2011;19:174-82.

9. González-Lucena G, Gelber PE, Pelfort X, Tey M, Monllau JC. Meniscal allograft transplantation without bone blocks: a 5-to 8-year follow-up of 33 patients. Arthroscopy. 2010;26:1633-40.

10. Chen MI, Branch TP, Hutton WC. Is it important to secure the horns during lateral meniscal transplantation? A cadaveric study. Arthroscopy. 1996;12: 174-81.

11. Paletta GA Jr, Manning T, Snell E, Parker R, Bergfeld J. The effect of allograft meniscal replacement on intraarticular contact area and pressures in the human knee. A biomechanical study. Am J Sports Med. 1997;25:692-8.
12. Kim JG, Lee YS, Lee SW, Kim YJ, Kong DH, Ko MS. Arthroscopically assisted medial meniscal allograft transplantation using a modified bone plug to facilitate passage: surgical technique. J Knee Surg. 2009;22:259-63.

13. McConkey M, Lyon C, Bennett DL, Schoch B, Britton C, Amendola A, et al. Radiographic sizing for meniscal transplantation using 3-D CT reconstruction. J Knee Surg. 2012;25:221-5.

14. Van Thiel GS, Verma N, Yanke A, Basu S, Farr J, Cole B. Meniscal allograft size can be predicted by height, weight, and gender. Arthroscopy. 2009;25:722-7.

15. Verdonk $P$, Vansintjan $P$, Verdonk R. Meniscal allograft transplantation. In: Bonnin M, Amendola NA, Bellemans J, MacDonald SJ, Menetrey J, editors. The knee joint: surgical techniques and strategies. Berlin Heidelberg: Springer; 2012. p. 139-47.

16. Johnson DL, Swenson TM, Livesay GA, Aizawa H, Fu FH, Harner CD. Insertion-site anatomy of the human menisci: gross, arthroscopic, and topographical anatomy as a basis for meniscal transplantation. Arthroscopy. 1995:11:386-94

17. Ha JK, Jang HW, Jung JE, Cho SI, Kim JG. Clinical and radiologic outcomes after meniscus allograft transplantation at 1-year and 4-year follow-up. Arthroscopy. 2014;30:1424-9.

18. Lee BS, Bin SI, Kim JM. Articular cartilage degenerates after subtotal/total lateral meniscectomy but radiographic arthrosis progression is reduced after meniscal transplantation. Am J Sports Med. 2016:44:159-65.

19. Ha JK, Sung JH, Shim JC, Seo JG, Kim JG. Medial meniscus allograft transplantation using a modified bone plug technique: clinical, radiologic, and arthroscopic results. Arthroscopy. 2011;27:944-50.

20. Wang H, Gee AO, Hutchinson ID, Stoner K, Warren RF, Chen TO, et al. Bone plug versus suture-only fixation of meniscal grafts: effect on joint contact mechanics during simulated gait. Am J Sports Med. 2014;42:1682-9.

21. Roberson TA, Wyland DJ. Meniscal allograft transplantation with soft tissue in bone socket fixation: arthroscopic technique with technical pearls. Arthrosc Tech. 2017;24(6):e483-9.

22. Ahn JH, Kang HW, Yang TY, et al. Multivariate analysis of risk factors of graft extrusion after lateral meniscus allograft transplantation. Arthroscopy. 2016; 32:1337-45

23. Lee BS, Kim HJ, Lee CR, et al. Clinical outcomes of meniscal allograft transplantation with or without other procedures: a systematic review and meta-analysis. Am J Sports Med. 2017:12:036354651772696.

24. Samitier G, Alentorn-Geli E, Taylor DC, Rill B, Lock T, Moutzouros V, et al. Meniscal allograft transplantation. Part 2: systematic review of transplant timing, outcomes, return to competition, associated procedures, and prevention of osteoarthritis. Knee Surg Sports Traumatol Arthrosc. 2015;23:323-33.

25. Barber-Westin SD, Noyes FR. Low-impact sports activities are feasible after meniscus transplantation: a systematic review. Knee Surg Sports Traumato Arthrosc. 2017. https://doi.org/10.1007/s00167-017-4658-8.

26. Cavanaugh JT. Rehabilitation of meniscal injury and surgery. J Knee Surg. 2014:27:459-78.

27. Myers P, Tudor F. Meniscal allograft transplantation: how should we be doing it? A systematic review. Arthroscopy. 2015:31:911-25.

\section{Submit your next manuscript to BioMed Central and we will help you at every step:}

- We accept pre-submission inquiries

- Our selector tool helps you to find the most relevant journal

- We provide round the clock customer support

- Convenient online submission

- Thorough peer review

- Inclusion in PubMed and all major indexing services

- Maximum visibility for your research

Submit your manuscript at www.biomedcentral.com/submit
Biomed Central 